\title{
De novo variants in an extracellular matrix protein coding gene, fibulin-5 (FBLN5) are associated with pseudoexfoliation
}

\author{
Biswajit Padhy ${ }^{1,2} \cdot$ Ramani Shyam Kapuganti ${ }^{1,2} \cdot$ Bushra Hayat $^{1,2} \cdot$ Pranjya Paramita Mohanty $^{3} \cdot$ \\ Debasmita Pankaj Alone $\mathbb{B}^{1,2}$
}

Received: 28 August 2018 / Revised: 5 July 2019 / Accepted: 16 July 2019 / Published online: 29 July 2019

(c) The Author(s), under exclusive licence to European Society of Human Genetics 2019

\begin{abstract}
Fibulin-5 (FBLN5), an extracellular scaffold protein, plays a crucial role in the activation of Lysyl oxidase like-1 (LOXL1), a tropoelastin crosslinking enzyme, and subsequent deposition of elastin in the extracellular matrix. Following study identifies polymorphisms within FBLN5 gene as risk factors and its aberrant expression in the pathogenesis of an ocular disorder, pseudoexfoliation (PEX). Exons and exon-intron boundaries within FBLN5 gene were scanned through fluorescence-based capillary electrophoresis for polymorphisms as risk factors for PEX pathogenesis in recruited study subjects with Indian ethnicity. mRNA and protein expression of FBLN5 was checked in lens capsule of study subjects through qRT-PCR and western blotting, respectively. In vitro functional analysis of risk variants was done through luciferase reporter assays. Thirty study subjects from control and PEX affected groups were scanned for potential risk variants. Putative polymorphisms identified by scanning were further evaluated for genetic association in a larger sample size comprising of 338 control and 375 PEX affected subjects. Two noncoding polymorphisms, hg38 chr14: g.91947643G>A (rs7149187:G>A) and hg38 chr14:g.91870431T>C (rs929608:T>C) within FBLN5 gene are found to be significantly associated with PEX as risk factors with a $p$-value of 0.005 and 0.004 , respectively. Molecular assays showed a decreased expression of FBLN5 at both mRNA and protein level in lens capsule of pseudoexfoliation syndrome (PEXS) affected subjects than control. This study unravels two novel risk variants within FBLN5 gene in the pathogenesis of PEX. Further, a decreased expression of FBLN5 in PEXS affected lens capsules implicates a pathogenic link between extracellular matrix maintenance and onset of PEX.
\end{abstract}

Supplementary information The online version of this article (https:// doi.org/10.1038/s41431-019-0482-6) contains supplementary material, which is available to authorized users.

$\triangle$ Pranjya Paramita Mohanty

drpranjya@gmail.com

$\triangle$ Debasmita Pankaj Alone

debasmita@niser.ac.in

1 National Institute of Science Education and Research, Bhubaneswar, Odisha 752050, India

2 Homi Bhabha National Institute, Training School Complex, Anushakti Nagar, Mumbai 400094, India

3 Sri Sri Borda Hospital, Dhauli, Bhubaneswar, Odisha 751002, India

\section{Introduction}

Pseudoexfoliation (PEX [MIM: 177650]) is an age-related ocular disorder and is diagnosed by deposition of proteinaceous aggregates called PEX fibrils on the tissue surface of anterior eye segment [1]. This initial stage of PEX is called as pseudoexfoliation syndrome (PEXS). In the later severe form of PEX, degeneration of retinal ganglion cell axons in the optic nerve head $(\mathrm{ONH})$ region located in the posterior segment of the eye is seen and is known as pseudoexfoliation glaucoma (PEXG) [1]. PEX is a leading cause of glaucoma worldwide [2]. Previous studies have done proteomic [3], immunostaining, and electron microscopic analysis $[4,5]$ of PEX fibrils, which shows that they are made up of extracellular protein aggregates arranged in a microfibrillar form. Genetic and biochemical studies have also shown the involvement of various genes coding for extracellular proteins, membrane proteins, as well as proteins involved in the unfolded protein response as risk 
factors in the pathogenesis of PEX, such as Lysyl oxidase like-1 (LOXL1) [6, 7], Clusterin [8-10], Contactinassociated protein-like 2 (CNTNAP2) [11], calcium voltage-gated channel subunit- $\alpha 1 \mathrm{~A}(C A C N A 1 A)$ [12], neuronal development, and actin remodeling proteins [13], Synoviolin1, and Calnexin [14]. However, low penetrance of reported genetic variants and inconsistent outcomes of previous studies across various ethnicities indicate the presence of other genetic factors in the etiology of PEX and the precise cause in the pathogenesis of PEX is still not clear.

In this study, we propose that Fibulin-5 (FBLN5 [MIM: 604580]), an extracellular scaffold protein might play a pivotal role in the pathogenesis of PEX. FBLN5 also known as developmental arteries and neural crest EGF-like protein (DANCE) plays a crucial role in the formation of elastic fibers in a process called elastogenesis [15], cell-matrix adhesion [16], regulation of cell growth [17], modulation of matrix proteases [18], and integrin-dependent regulation of reactive oxygen species (ROS) in the extracellular matrix (ECM) [19]. Most importantly, FBLN5 assists in the deposition of the de-aminating enzyme LOXL1 [MIM: 153456], a major risk factor for PEX and its activation by pro-peptide cleavage in the ECM. Activated LOXL1 then crosslinks tropoelastin monomers by lysyl-deamination following coacervation into elastic fibers [20]. FBLN5 has been associated with various elastinopathic disorders, such as pelvic organ prolapse (POP) [21], cutis laxa [22, 23], abdominal aortic aneurysm [24], Charcot-Marie-Tooth disease (CMT) [25], and age-related macular degeneration (ARMD) [26]. Aberrant expression and/or abnormal deposition of FBLN5 is seen in patients with such disorders and results in impaired elastic fiber development. Further, recent studies have related the pathogenesis of PEX with these elastinopathies, such as ARMD [27], POP [28], and abdominal aortic aneurysm [29, 30]. To add to this, Schlotzer-Schrehardt et al. have shown an impaired ECM, similar to that in elastinopathies with irregular deposition pattern of LOXL1 and elastin aggregates in PEX affected posterior eye tissues (lamina cribrosa and peripapillary sclera) through immunostaining [31]. A study on 3D culture of tenon fibroblasts from PEX donors also showed an aberrant deposition of FBLN5 in vitro [32]. Altogether, these studies indicate a possible involvement of FBLN5 in the formation of PEX aggregates and thereby in its progression. Additionally, mass spectrometric analysis has showed the presence of another fibulin family member protein, Fibulin-2 along with LOXL1 in PEX aggregates [3].

In the present study, we show that polymorphisms within FBLN5 are associated with PEX. Also, aberrant expression of FBLN5 in PEXS affected tissues suggests its involvement in PEX pathogenesis. Current finding establishes a novel link between an ECM protein, FBLN5 with PEX, an ocular elastinopathy.

\section{Materials and methods}

\section{Study subjects}

This study was approved by the ethics review board of National Institute of Science Education and Research, India and adhered to the tenets of the Declaration of Helsinki. All the participants underwent a detailed ocular examination, including slit lamp microscopy, ocular biometry, Goldman applanation tonometry, $+90 \mathrm{D}$ biomicroscopic fundus evaluation, and four-mirror gonioscopy. Cataract patients diagnosed with PEXS or PEXG in screening camps and outpatient departments were included for this study. Informed consent was obtained from all individual participants recruited for the study. Similarly, cataract patients without PEXS or PEXG matching the inclusion criteria were included as controls. Demographic features of the study subjects included in the study are presented in Table 1. Lens capsule tissues from study subjects were collected in RNAlater stabilization solution (Invitrogen, USA) or in liquid nitrogen during cataract surgery and stored at $-80^{\circ} \mathrm{C}$ until further use.

\section{DNA extraction, PCR, elution, and sequencing}

Peripheral blood $(4 \mathrm{ml})$ was collected from study subjects for genomic DNA extraction through phenol-chloroform method. Eleven sets of primers were designed to amplify 11 exons of FBLN5 gene and their surrounding exon-intron boundaries through PCR of 30 randomly selected, age and gender-matched individuals from control, PEXS, and PEXG groups as discovery set. Primers were designed by Primer-BLAST and their detailed information is presented in the Supplementary Table 1. PCR products were eluted through gel elution (QIAquick Gel Extraction Kit, QIAGEN, Hilden) and subsequently sequenced through

Table 1 Demographic and clinical features of study subjects included for genotyping of $F B L N 5$ variants

\begin{tabular}{lllllll}
\hline Subjects & $\begin{array}{l}\text { Sample size } \\
(n)\end{array}$ & \multicolumn{2}{l}{ Age (in years) } & & \multicolumn{2}{l}{ Sex } \\
& & $\begin{array}{l}\text { Mean } \pm \\
\text { SD }\end{array}$ & Range & & Male & Female \\
\hline $\begin{array}{l}\text { PEX } \\
\text { combined }\end{array}$ & 375 & $70.3 \pm 8.4$ & $41-90$ & 276 & 99 \\
PEXS & 280 & $71 \pm 8.2$ & $41-90$ & 196 & 84 \\
PEXG & 95 & $68 \pm 8.6$ & $47-88$ & 80 & 15 \\
Control & 338 & $64.4 \pm 9.8$ & $40-90$ & 181 & 157 \\
\hline
\end{tabular}


fluorescence-based capillary electrophoresis using BigDye Terminator v.3.1 cycle sequencing kit (Applied Biosystems, Austin, TX 78744, USA) on 3130xl Genetic Analyser (Applied Biosystem) platform. Detailed procedures of PCR and sequencing of targeted product were followed as described previously [10]. Sequencing analysis software v5.3 (Applied Biosystem) and BioEdit v7.1 (http://www. mbio.ncsu.edu/bioedit/bioedit.html) was used for sequence analysis.

\section{RNA extraction, CDNA synthesis, and quantitative real time-PCR}

Total RNA was isolated from lens capsule tissues by using a RNA extraction kit (RNeasy Mini Kit, QIAGEN GmbH, Hilden). After RNA quality check, cDNA was synthesized from $100 \mathrm{ng}$ of total RNA through a Reverse Transcription Kit (Verso cDNA Synthesis Kit-AB1453A; Thermo Fisher Scientific). PrimerQuest Tool (IDT) was used to design gene-specific primers overlapping exon-exon junction for the mRNA transcript of FBLN5 with RefSeq ID NM_006329.3 and of GAPDH with RefSeq ID NM_002046.5 (Supplementary Table 1). qRT-PCR was performed using 7500 Real time PCR System (Applied Biosystems, Foster city, CA, USA). Total $5 \mathrm{ng}$ of cDNA and $0.4 \mu \mathrm{M}$ each of forward and reverse primers were used per $20 \mu \mathrm{l}$ reaction volume in triplicate for both FBLN5 and $G A P D H$. Amplification specificity of the PCR product was checked via melt curve analysis and sequencing. ${ }^{\Delta \Delta} \mathrm{Ct}$ method was used to calculate the expression change in fold for target gene and represented as fold difference in comparison to control. For normalization of FBLN5 gene expression, GAPDH expression was taken as an endogenous control.

\section{Protein extraction and western blotting}

Tissue extract from lens capsule tissues was prepared using NE-PER kit (Thermo Fisher Scientific) and quantified by Bradford protein assay (Sigma). Denatured protein extract $(15 \mu \mathrm{g})$ was then loaded on a $12 \%$ SDS-PAGE and subsequently transferred onto a PVDF membrane at $15 \mathrm{~V}$ for 45 min (Immobilion-P PVDF from Merck Millipore, Billerica, MA, USA). After blocking the membrane with 5\% skim milk, primary mouse monoclonal antibody against FBLN5 protein (ab66339; Abcam) was used in 1:200 dilution along with blocking solution. HRP-conjugated goat anti-mouse IgG (621140680011730, GeNei, India) was used as secondary antibody (1:5000 dilution). GAPDH antibody (ABM22C5, Abgenex, India) was used as an endogenous control. A chemiluminescence kit (SuperSignal $^{\mathrm{TM}}$ West Femto Maximum Sensitivity Substrate, Thermo scientific) was used for detection of bands and signals were detected in a Fusion Solo S Chemi-Doc (Vilber Lourmat, Eppendorf, Germany). Band intensity was quantified in Evolution Capt software.

\section{Cell culture}

The human cell line, HEK293 (National Center for Cell Science, Pune) was cultured in HiGlutaXL Dulbecco's modified Eagle medium, high glucose (AL007G, HiMedia, India) with $10 \%$ fetal bovine serum (RM9952, HiMedia). Human lens epithelial (HLE) cell line was procured from ATCC (B-3 CRL-11421, Virginia, USA) and cultured in DMEM/F12 medium (11330057, Invitrogen) and supplemented with $10 \%$ inactivated FBS (16000044, Invitrogen). All media were supplemented with $1 \%$ penicillin $(100 \mathrm{U} /$ $\mathrm{ml})$ and streptomycin $(0.1 \mathrm{mg} / \mathrm{ml})$ (A001, HiMedia) and maintained at $37^{\circ} \mathrm{C}$ and $5 \% \mathrm{CO}_{2}$.

\section{Luciferase reporter assays}

Luciferase reporter assays were performed as mentioned previously [33]. pGL4.23 luciferase vector and pGL4.74 renilla vector were used for reporter assays (Promega, Madison, WI, USA). A genomic region of $200 \mathrm{bp}$ surrounding hg38 chr14:g.91947643G $>$ A (rs7149187:G>A) harboring " $\mathrm{A}$ " or " $\mathrm{G}$ " and $176 \mathrm{bp}$ surrounding hg38 chr14:g.91870431T>C (rs929608:T>C) harboring "C" or "T" variant, respectively, was PCR-amplified using two sets of specific primer pair (Supplementary Table 1) from the extracted genomic DNA of study subjects. The amplified products were then cloned into pGL4.23 vector by double digestion and subsequent ligation at KpnI-HF and XhoI-HF sites (New England Biolabs, Ipswich, MA). Luciferase constructs $(1 \mu \mathrm{g})$ and renilla vector $(10 \mathrm{ng})$ were co-transfected (Lipofectamine 2000, Invitrogen) into HEK293 and HLE cells individually, and seeded in a 12well plate at $80 \%$ confluence. Dual-Luciferase ${ }^{\circledR}$ Reporter Assay System (Promega) was used to measure the reporter activity in a Varioskan Flash Multimode reader (Thermo Fisher Scientific, Waltham, MA, USA) in the prepared cell lysate after $24 \mathrm{~h}$ of post-transfection. Luciferase activity was analyzed after normalizing with renilla reporter activity for transfection efficiency. Each of the experiments were repeated independently with at least three replicates.

\section{Genetic and statistical analyses}

Allelic association tests, Hardy-Weinberg equilibrium (HWE), Bonferroni correction, and correction for multiple testing with permutation analysis $(n=10,000)$ were done by PLINK. Effect of confounding factors, age, and sex were eliminated through logistic regression model using PLINK. 
Table 2 Distribution of FBLN5 variants in PEX and control subjects

\begin{tabular}{|c|c|c|c|c|c|c|c|}
\hline SNP & Major allele & Minor allele & MAF in control $(n=338)$ & MAF in PEX combined $(n=375)$ & $p$-value & $p$-value ${ }^{\dagger}$ & OR $(95 \% \mathrm{CI})$ \\
\hline rs7149187 & G & A & 0.39 & 0.31 & 0.005 & 0.019 & $0.72(0.57 \pm 0.91)$ \\
\hline rs2430347 & G & A & 0.23 & 0.23 & 0.77 & 0.99 & $1.03(0.8 \pm 1.34)$ \\
\hline rs929608 & $\mathrm{T}$ & $\mathrm{C}$ & 0.53 & 0.45 & 0.004 & 0.017 & $0.73(0.58 \pm 0.9)$ \\
\hline SNP & Major allele & Minor allele & MAF in control $(\boldsymbol{n}=338)$ & MAF in PEXS $(\boldsymbol{n}=280)$ & $p$-value & $p$-value ${ }^{\dagger}$ & OR $(95 \% \mathrm{CI})$ \\
\hline rs7149187 & G & A & 0.39 & 0.32 & 0.02 & 0.06 & $0.74(0.58 \pm 0.95)$ \\
\hline rs2430347 & G & A & 0.23 & 0.25 & 0.48 & 0.85 & $1.1(0.83 \pm 1.45)$ \\
\hline rs929608 & $\mathrm{T}$ & $\mathrm{C}$ & 0.53 & 0.45 & 0.007 & 0.02 & $0.72(0.57 \pm 0.91)$ \\
\hline SNP & Major allele & Minor allele & MAF in control $(n=338)$ & MAF in PEXG $(\boldsymbol{n}=95)$ & $p$-value & $p$-value ${ }^{\dagger}$ & OR $(95 \% \mathrm{CI})$ \\
\hline rs7149187 & $\mathrm{G}$ & A & 0.39 & 0.29 & 0.02 & 0.07 & $0.66(0.46 \pm 0.94)$ \\
\hline rs2430347 & G & A & 0.23 & 0.2 & 0.48 & 0.86 & $0.86(0.58 \pm 1.29)$ \\
\hline rs929608 & $\mathrm{C}$ & $\mathrm{T}$ & 0.47 & 0.54 & 0.08 & 0.24 & $1.33(0.96 \pm 1.86)$ \\
\hline
\end{tabular}

Frequency of minor allele "A" at rs7149187 in control (0.39) is significantly higher in comparison to PEX $(0.31, p=0.005)$, PEXS $(0.32, p=$ $0.02)$, and PEXG $(0.29, p=0.02)$ individuals. Similarly, frequency of minor allele "C" at rs929608 is also found to be significantly higher in control $(0.53)$ than in PEX $(0.45, p=0.004)$ and PEXS $(0.45, p=0.007)$ but not in PEXG $(0.46, p=0.08)$ individuals. Risk analysis of minor alleles was done to calculate the odds ratio based on an Allelic model

$n$ sample size, $M A F$ minor allele frequency, $O R$ odds ratio, $C I$ confidence interval

${ }^{\dagger} p$-value after permutation correction, where $n=10,000$

Haplotype analysis and linkage disequilibrium (LD) analysis were done using Haploview V4.2. Online Genetic Power Calculator (zzz.bwh.harvard.edu/gpc/) was used to calculate statistical power of the association study. Statistical significance of groupwise results was analyzed through Student's $t$-test and $p<0.05$ was considered as statistically significant. All experiments were repeated at least three times. Descriptive data were presented as mean \pm SEM. The data for the genetic association study is available at https:// databases.lovd.nl/shared/individuals/FBLN5 with individuals IDs: 00226182 (rs7149187-homozygous mutant [AA]-controls), 00235387 (rs7149187-homozygous mutant [AA]-PEX), 00226181 (rs7149187-heterozygous [AG]controls), 00228154 (rs7149187-heterozygous [AG]-PEX), 00235390 (rs2430347-homozygous mutant [GG]-controls), 00235394 (rs2430347-homozygous mutant [GG]-PEX), 00235389 (rs2430347-heterozygous [GA]-controls), 00235393 (rs2430347-heterozygous [GA]-PEX), 00235398 (rs929608-homozygous mutant [CC]-controls), and 00235396 (rs929608-homozygous mutant [CC]-PEX), 00235397 (rs929608-heterozygous [CT]-controls), 00235395 (rs929608-heterozygous [CT]-PEX).

\section{Results}

\section{FBLN5 variants, rs7149187:G>A and rs929608:T>C are genetically associated with PEX}

As a discovery set, we sequenced 11 exons and the exon-intron boundaries of FBLN5 gene in 30 age and sexmatched PEX affected cases and 30 controls. We found a synonymous nucleotide variant, rs2430347:A>G (hg38 chr14:g.91881336A $>$ G) in the 9th exon and two non-coding variants, rs7149187:G>A (hg38 chr14:g.91947643G>A) and rs929608:T>C (hg38 chr14:g.91870431T>C) in the 5' untranslated region (UTR) and 10th intron, respectively. In the subsequent replicative set, these three polymorphisms were checked for genetic association comprising of 338 controls and 375 PEX cases (PEXS-280 and PEXG-95). Prior to genetic analysis, allele frequencies of SNPs were checked for HWE at a default significance threshold $(p \leq 0.001)$ and SNPs rs929608, rs2430347, and rs7149187 passed HWE test with a $p$-value of $0.75,0.32$, and 0.01 , respectively. Distribution of allele frequency, statistical significance, and odds ratio for each of these SNPs are presented in Table 2. Both rs7149187 ( $p=0.005)$ and rs929608 $(p=0.004)$ were found to be significantly associated with PEX with risk allele " $G$ " and "T", respectively. However, rs2430347 ( $p=0.77)$ was not found to be significantly associated with PEX. After Bonferroni correction for multiple comparisons, both rs7149187 and rs929608 retained their significance with a $p$ value of 0.016 and 0.014 , respectively. Also, after correction for multiple testing with permutation analysis $(n=10,000)$ both rs7149187 and rs929608 remained significant with a $p$ value of 0.019 and 0.017 , respectively. After correction for covariates (age and sex) both SNPs rs7149187 and rs929608 remained significantly associated with PEX with a $p$-value of 0.009 and 0.004 , respectively. Genotypic distribution of variants and analysis are presented in Supplementary Table 2.

Genetic analysis of polymorphisms was also done after segregation of PEX into PEXS and PEXG groups. While rs7149187 was found to be significant in both PEXS $(p=0.02)$ and PEXG $(p=0.023)$ individuals, rs929608 remained 
Table 3 Haplotype association of the FBLN5 variants with PEX

\begin{tabular}{llll}
\hline Risk haplotype "T-G-G" (rs929608-rs2430347-rs7149187) & Haplotype freq. & $p$-value & $p$-value \\
\hline Control & 0.22 & - & - \\
PEX combined & 0.28 & 0.007 & 0.03 \\
PEXS & 0.27 & 0.03 & 0.13 \\
PEXG & 0.30 & 0.01 & 0.05 \\
\hline
\end{tabular}

Frequency of risk haplotype T-G-G (rs929608-rs2430347-rs7149187) in control (0.22) is significantly lower in comparison to PEX $(0.28, p=0.007)$, PEXS $(0.27, p=0.03)$, and PEXG $(0.3, p=0.01)$ individuals ${ }^{\dagger} p$-value after permutation correction where $n=10,000$ significant only with PEXS $(p=0.007)$ but not with PEXG individuals $(p=0.08)$ (Table 2). Similar to that of PEX combined group, rs2430347 was not found to be significant in either PEXS $(p=0.47)$ or PEXG $(p=0.48)$ groups. Risk analysis based on an allelic model showed that minor allele "A" at rs7149187 confers a protective effect with an odds ratio (OR) of 0.72 for PEX, 0.74 for PEXS, and 0.66 for PEXG. Similarly, minor allele "C" at rs929608 confers an OR of 0.73 for PEX and 0.72 for PEXS individuals. As evident from the LD value $\left(D^{\prime}=0.02\right.$, LOD score $=0.03, r$-squared $\left.=0\right)$ there is no linkage between the markers rs929608 and rs7149187. As shown in Table 3, haplotype analysis showed that frequency of risk haplotype "T-G-G" (rs929608-rs2430347-rs7149187) is significantly higher in PEX combined (0.28, $p=0.007)$, PEXS $(0.27, p=0.03)$, and PEXG $(0.3, p=0.01)$ groups in comparison to control group $(0.22)$.

\section{Downregulation of FBLN5 mRNA and protein expression in PEXS affected tissues}

Subsequently we checked the mRNA expression of the single reported transcript variant of FBLN5 gene (NM_006329.3) in the lens capsule of control and cases through qRT-PCR. As shown in Fig. 1a, FBLN5 expression was found to be half-fold downregulated in PEXS-affected individuals $(0.53$-fold, $p=0.04)$ than that of control subjects (1.0 fold). However, we did not find a significant difference between PEXG (0.73-fold, $p=0.34$ ) and control group. Western blot analysis of total cell lysate extracted from lens capsules by using a previously reported [34, 35] c-terminus-specific FBLN5 antibody showed a prominent band at $\sim 55 \mathrm{kDa}$ in study subjects. We found a significantly $(p=0.04)$ decreased expression of FBLN5 in PEXS subjects than in control as shown through western (Fig. 1b, c). However, there is no difference $(p=0.36)$ in the level of FBLN5 between control and PEXG subjects (Fig. 1b, c).

\section{Risk variants at both rs7149187:G>A and rs929608: $T>C$ lack a functional significance}

Suspecting an allele-based regulatory role, we hypothesized that the genomic region surrounding associated variants may act as a regulator (enhancer/suppressor) for FBLN5 gene expression. To validate this, luciferase reporter assays were carried out by cloning a $200 \mathrm{bp}$ genomic region surrounding rs7149187 (either " $\mathrm{A}$ " or "G") or $176 \mathrm{bp}$ surrounding rs929608 (either "T" or "C") upstream into the minimal promoter of luciferase vector, pGL4.23 and transfected into both HEK293 and HLE cells. As shown in Fig. 2a, transition from allele " $\mathrm{A}$ " to allele " $\mathrm{G}$ " at rs7149187 did not change the luciferase activity in either HEK293 $(p=0.3)$ or HLE $(p=0.21)$ cells. Similarly, constructs containing rs929608 allelic variant " $C$ " or "T" did not show any difference in reporter activity in both HEK293 $(p=0.48)$ or HLE cells $(p=0.06)$ as shown in Fig. 2b. However, we found a significant difference in luciferase expression between control empty vector and constructs with either alleles at rs7149187 (Fig. 2a) or rs929608 (Fig. 2b) in both HEK293 and HLE cells. This regulatory effect can be attributed to the genomic region surrounding the variants used for cloning suggesting other regulatory mechanisms rather than the SNPs itself. Similarly, a genotype-correlated expression analysis does not show an allele-dependent effects at both of the variants on FBLN5 gene expression in lens capsule tissues (Supplementary Fig. 2a and b).

\section{Discussion}

FBLN5 (Gene ID: 10516) is an extracellular secreted protein and belongs to class II fibulin subfamily with shorter repeats of calcium-binding epidermal growth factor like motifs (cbEGF) unlike that of class I subfamily with long repeats. It is secreted as a $66-\mathrm{kDa}$ protein containing 425 amino acids from a wide variety of cells including fibroblast and vascular smooth muscle cells [36]. After secretion, a conserved RGD motif (Arginine-Glycine-Aspartate) present in the N-terminus of FBLN5 binds to integrin on the cell surface and is essential for its cellular function. Similarly, C-terminus of FBLN5 plays a key role in elastogenesis by interacting with N-terminus of LOXL1, which afterwards assists in polymerization and deposition of tropoelastin monomers into elastin fibrils [15]. Deregulation of FBLN5 has been reported in various disorders involving impaired 


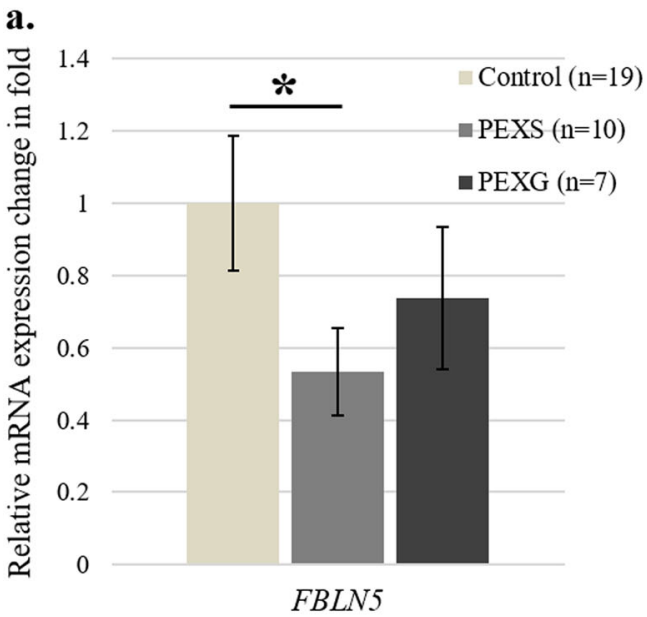

b.

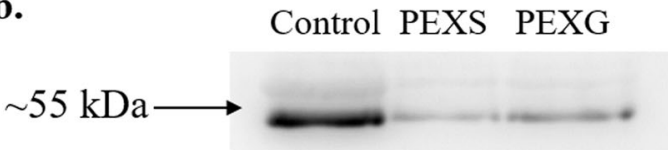

$\sim 35 \mathrm{kDa} \longrightarrow$

Fig. 1 mRNA and protein expression of FBLN5 in the lens capsule of cases and controls. a FBLN5 expression was found to be significantly downregulated in PEXS $(0.53 \pm 0.1)$ compared to that of control $(1 \pm$ $0.1)$ with a $p$-value of 0.04 but not in PEXG subjects $(0.73 \pm 0.1, p=$ 0.34). b Represents immunoblots for FBLN5 protein expression in the lens capsule of control versus PEXS and PEXG subjects. c Quantitative analysis of FBLN5 protein in control versus PEXS and PEXG subjects. Compared to lens capsule of control $(1 \pm 0.2)$, in PEXS $(0.26$

maintenance of ECM. Also, common genomic variations within FBLN5 gene have been associated with various ECM disorders. Since, PEX involves abnormal deposition and impaired maintenance of ECM proteins we hypothesized that FBLN5 can be a genetic risk factor in the etiology of PEX.

\section{Variants within FBLN5 are risk factors for PEX pathogenesis}

Previous studies have reported genetic polymorphisms within FBLN5 as risk factors for diseases, such as ARMD [26, 37], cutis laxa [37-39], POP [40], and CMT [41]. A study by Auer-Grumbach et al. linked missense variations in FBLN5 with concurrent pathological alterations seen in ARMD, CMT, and hyperelastic skin [42]. Similarly, various synonymous and nonsynonymous variations in FBLN5 were found in a proband individual suffering from cutis laxa [23]. Further, reports suggest that missense variants within FBLN5 that are associated with ARMD and cutis laxa leads to misfolding, decreased secretion, increased c.

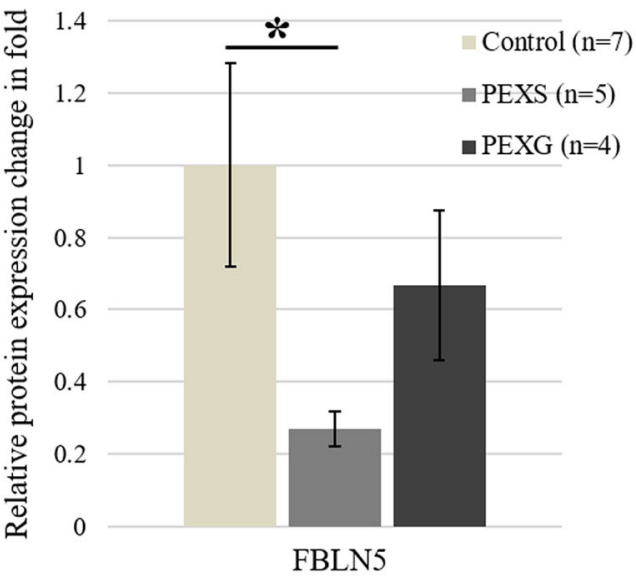

FBLN5

\section{GAPDH}

$\pm 0.04)$ subjects there is a significantly decreased $(p=0.04)$ FBLN5 expression. However, there is no significant $(p=0.36)$ difference in the FBLN5 protein level between control and PEXG $(0.66 \pm 0.2)$ subjects. Sample size is denoted by " $n$ " and relative expression change in fold is represented as mean \pm SEM. Each experiment is repeated at least three times. Student's $t$-test was used to calculate the statistical significance between groups, $* p<0.05$

aggregation of FBLN5, and incomplete ECM formation $[22,37,43,44]$.

We did not find any coding DNA sequence variation in PEX-affected individuals. However, in discovery set we found three genetic polymorphisms in the FBLN5 gene (rs7149187, rs2430347, and rs929608), which were later included in the replicative stage for statistical association. We found a significant association of two noncoding variants, hg38 chr14:g.91947643G >A (rs7149187:G>A) and hg38 chr14:g.91870431T>C (rs929608:T>C) within $F B L N 5$, as risk factors for PEX pathogenesis. Previously, rs929608 has been picked as a risk factor for ARMD in a case-control study conducted in Indian population [45] and in a recessive Iranian cutis laxa pedigree by Elahi et al. [23]. Further, epidemiological association of ARMD with PEX corroborates the role of rs929608 thereby, FBLN5 in the pathogenesis of these disorders $[27,46]$. In the past, studies have reported the role of PEX-associated non-coding genetic variants in genes, such as LOXL1 [47, 48] and Clusterin $[10,33]$, playing a role in PEX pathogenesis by altering their gene expression. However, functional in vitro 
a.

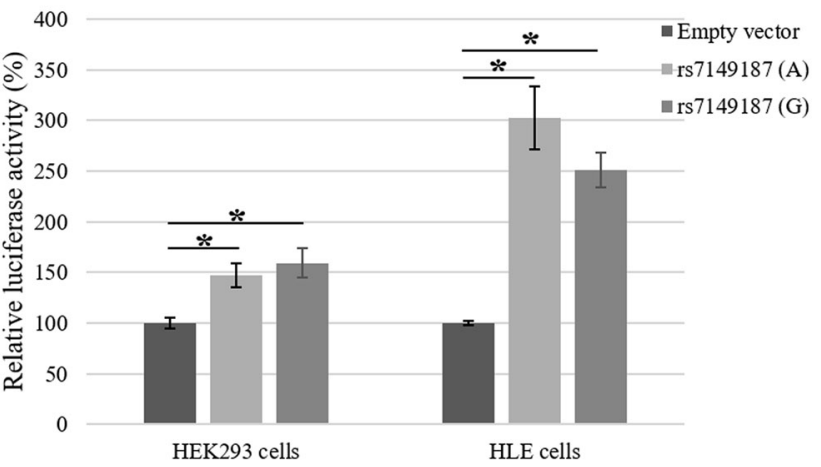

b.

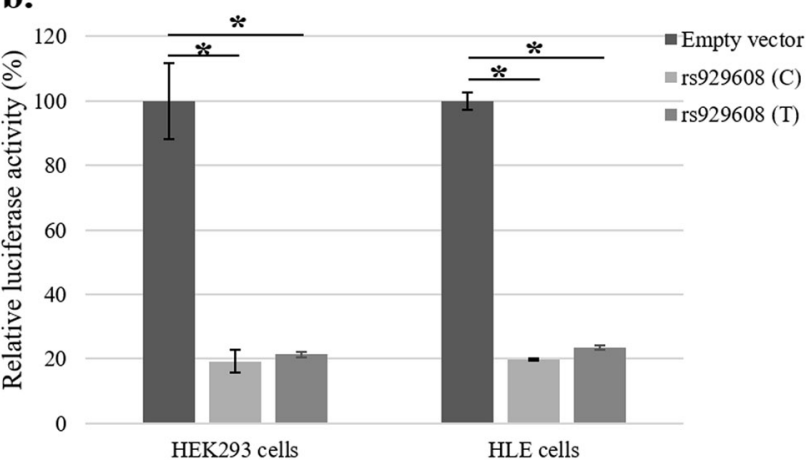

Fig. 2 Effect of allele changes at rs7149187 and rs929608 on luciferase activity. a Related luciferase activity is shown for empty reporter vector and constructs containing rs 7149187 with either "A" or "G" alleles. There is a significant difference in reporter activity in HEK293 cells between control empty vector $(100 \pm 5)$ and "A" (147 \pm $11)$ or "G" $(159 \pm 14)$ alleles with $p$-values of 0.008 and 0.01 , respectively. However, there is no difference in luciferase expression between the alleles $(p=0.3)$. Similarly, in HLE cells the difference between empty vector $(100 \pm 2)$ and "A" $(302 \pm 31)$ or " $G$ " $(251 \pm 17)$ alleles were shown to be significant ( $p=0.02$ and 0.01 , respectively) but no significant difference was observed between the alleles $(p=$ 0.21 ). b Relative luciferase activity is shown for empty vector and constructs containing rs929608 with either "C" or " $\mathrm{T}$ " alleles. Reporter activity is significantly different between empty vector $(100 \pm 11)$ and "C" $(19 \pm 3)$ or "T" $(21 \pm 0.9)$ alleles with $p$-value of 0.007 and 0.01 , respectively in HEK293 cells but not within "C" or "T" constructs ( $p$ $=0.48$ ). Similarly, in HLE cells the difference between empty vector $(100 \pm 2)$ and both "C" $(19 \pm 0.4)$ or "T" $(23 \pm 0.7)$ alleles were shown to be significant with a $p$-value of 0.002 and 0.001 , respectively; but there was no difference between " $\mathrm{C}$ " and " $\mathrm{T}$ " alleles $(p=0.06)$. Each experiment is repeated at least three times. Relative activity is represented as mean \pm SEM. Student's $t$-test was used to calculate the statistical significance between groups, ${ }^{*} p<0.05$

reporter assays and genotype-correlated tissue expression analysis carried out in the study, as well as in silico analysis done through Encyclopedia of DNA elements (ENCODE) and Genotype-Tissue Expression (GTEx) suggests, both rs7149187 and rs929608 does not have regulatory role over FBLN5 gene expression. Further studies are being undertaken to find the role of nearby functional SNPs and influence of epigenetic factors as causative factors in PEX pathogenesis.
Decreased FBLN5 expression may lead to abnormal maintenance of ECM

Aberrant expression of FBLN5 has been seen in many ECM disorders like ARMD [37, 49], cutis laxa [22, 37], and POP $[21,50,51]$. Diminished level of FBLN5 in the ECM results in impaired elastic fiber development by a reduced interaction with other extracellular proteins like LOXL1, elastin, and fibrillin-1, and is the sole factor in the progression of such diseases. Also, deregulated expression of FBLN5 is seen in different cancerous tissues [52, 53]. Chen et al. have shown that FBLN5 acts as a metastasis suppressor in lung cancer by inhibiting Wnt/B-catenin pathway thereby reducing matrix metalloproteinase-7 (MMP-7) [52]. Further, it also stimulates integrin-induced production of ROS which then inhibits tumor growth [19].

We found a significantly decreased expression of FBLN5 in PEXS-affected lens capsule tissues. Similar to FBLN5, aberrant expression of other ECM-related proteins, such as LOXL1 [54], Fibrillin-1 [55, 56], and elastin [31] were shown in PEX-affected eye tissues. Of these, LOXL1, a key ECM maintaining enzyme and an interacting partner of FBLN5 is reported to be the major risk factor in PEX pathogenesis [6, 47]. After binding to FBLN5, LOXL1 activates and cross-links tropoelastin molecules into mature elastic fibers. However, in PEX-affected tissues LOXL1 was not found to be co-localized with FBLN5 as in normal cases [57]. This suggests a loss of interaction between LOXL1 and FBLN5 at the site of PEX material formation. Further, studies have shown that decreased expression or knock-out of FBLN5 leads to irregular deposition of inactive LOXL1 in large aggregates along with its substrate elastin in the mouse dermis [58], a feature similar to that seen in PEX-affected posterior eye tissues [31]. Additionally, Hirai et al. have shown that the amount of active FBLN5 diminishes with age [59] with a decrease in ECM maintenance, which leads to impaired ECM in aged tissues and may aggravate the disease condition in ageing elastinopathies and might as well in PEXS. However, unlike PEXS, reduced expression of FBLN5 in PEXG-affected tissues were not significantly different than control. This may implicate an involvement of other epigenetic factors regulating FBLN5 expression at different stages of the disease and further studies need to be done to elucidate the molecular mechanism through which FBLN5 imparts a role in PEX development.

In summary, current work establishes a novel association between genetic variants in FBLN5 with PEX suggesting $F B L N 5$ as a contributing factor in the disease pathogenesis. Further, decreased expression of FBLN5 in lens capsule of PEXS-affected tissues implicates an impaired maintenance of the ECM protein in PEX development. However, finding of functional genetic risk variants, if any and the adverse 
effect of decreased FBLN5 expression during the onset of PEX remains to be explored.

Acknowledgements The authors thank the study participants for their contribution and consent for this study. This work was supported by the National Institute of Science Education and Research, Department of Atomic Energy (India); and Council of Scientific and Industrial Research (India) Grant no. 27(0317)/16/EMR-II.

\section{Compliance with ethical standards}

Conflict of interest The authors declare that they have no conflict of interest.

Publisher's note: Springer Nature remains neutral with regard to jurisdictional claims in published maps and institutional affiliations.

\section{References}

1. Schlotzer-Schrehardt U, Naumann GO. Ocular and systemic pseudoexfoliation syndrome. Am J Ophthalmol 2006;141:921-37.

2. Ritch R. Exfoliation syndrome-the most common identifiable cause of open-angle glaucoma. J Glaucoma 1994;3:176-7.

3. Ovodenko B, Rostagno A, Neubert TA, Shetty V, Thomas S, Yang A, et al. Proteomic analysis of exfoliation deposits. Invest Ophthalmol Vis Sci 2007;48:1447-57.

4. Li ZY, Streeten BW, Wallace RN. Association of elastin with pseudoexfoliative material: an immunoelectron microscopic study. Curr Eye Res 1988;7:1163-72.

5. Schlotzer-Schrehardt U, Dorfler S, Naumann GO. Immunohistochemical localization of basement membrane components in pseudoexfoliation material of the lens capsule. Curr Eye Res 1992;11:343-55.

6. Thorleifsson G, Magnusson KP, Sulem P, Walters GB, Gudbjartsson DF, Stefansson $\mathrm{H}$, et al. Common sequence variants in the LOXL1 gene confer susceptibility to exfoliation glaucoma. Science 2007;317:1397-400.

7. Aung T, Ozaki M, Lee MC, Schlotzer-Schrehardt U, Thorleifsson G, Mizoguchi T, et al. Genetic association study of exfoliation syndrome identifies a protective rare variant at LOXL1 and five new susceptibility loci. Nat Genet 2017;49:993-1004.

8. Krumbiegel M, Pasutto F, Mardin CY, Weisschuh N, Paoli D, Gramer E, et al. Exploring functional candidate genes for genetic association in german patients with pseudoexfoliation syndrome and pseudoexfoliation glaucoma. Invest Ophthalmol Vis Sci 2009;50:2796-801.

9. Burdon KP, Sharma S, Hewitt AW, McMellon AE, Wang JJ, Mackey DA, et al. Genetic analysis of the clusterin gene in pseudoexfoliation syndrome. Mol vision 2008;14:1727-36.

10. Padhy B, Nanda GG, Chowdhury M, Padhi D, Rao A, Alone DP. Role of an extracellular chaperone, clusterin in the pathogenesis of pseudoexfoliation syndrome and pseudoexfoliation glaucoma. Exp Eye Res 2014;127:69-76.

11. Krumbiegel M, Pasutto F, Schlotzer-Schrehardt U, Uebe S, Zenkel M, Mardin CY, et al. Genome-wide association study with DNA pooling identifies variants at CNTNAP2 associated with pseudoexfoliation syndrome. Eur J Hum Genet 2011;19:186-93.

12. Aung T, Ozaki M, Mizoguchi T, Allingham RR, Li Z, Haripriya $\mathrm{A}$, et al. A common variant mapping to CACNA1A is associated with susceptibility to exfoliation syndrome. Nat Genet 2015;47:387-92.

13. Zagajewska K, Piatkowska M, Goryca K, Balabas A, Kluska A, Paziewska A, et al. GWAS links variants in neuronal development and actin remodeling related loci with pseudoexfoliation syndrome without glaucoma. Exp Eye Res 2018;168:138-48.

14. Hayat B, Padhy B, Mohanty PP, Alone DP. Altered unfolded protein response and proteasome impairment in pseudoexfoliation pathogenesis. Exp Eye Res 2019;181:197-207.

15. Liu X, Zhao Y, Gao J, Pawlyk B, Starcher B, Spencer JA, et al. Elastic fiber homeostasis requires lysyl oxidase-like 1 protein. Nat Genet 2004;36:178-82.

16. Tang JC, Liu JH, Liu XL, Liang X, Cai XJ. Effect of fibulin-5 on adhesion, migration and invasion of hepatocellular carcinoma cells via an integrin-dependent mechanism. World J Gastroenterol 2015;21:11127-40.

17. Lomas AC, Mellody KT, Freeman LJ, Bax DV, Shuttleworth CA, Kielty CM. Fibulin-5 binds human smooth-muscle cells through alpha5beta1 and alpha4beta1 integrins, but does not support receptor activation. Biochem J 2007;405:417-28.

18. Budatha M, Roshanravan S, Zheng Q, Weislander C, Chapman SL, Davis EC, et al. Extracellular matrix proteases contribute to progression of pelvic organ prolapse in mice and humans. J Clin Invest 2011;121:2048-59.

19. Schluterman MK, Chapman SL, Korpanty G, Ozumi K, Fukai T, Yanagisawa $\mathrm{H}$, et al. Loss of fibulin-5 binding to betal integrins inhibits tumor growth by increasing the level of ROS. Dis Model Mech 2010;3:333-42.

20. Thomassin L, Werneck CC, Broekelmann TJ, Gleyzal C, Hornstra IK, Mecham RP, et al. The Pro-regions of lysyl oxidase and lysyl oxidase-like 1 are required for deposition onto elastic fibers. J Biol Chem 2005;280:42848-55.

21. Jung HJ, Jeon MJ, Yim GW, Kim SK, Choi JR, Bai SW. Changes in expression of fibulin-5 and lysyl oxidase-like 1 associated with pelvic organ prolapse. Eur J Obstet Gynecol Reprod Biol 2009; 145:117-22.

22. Claus S, Fischer J, Megarbane H, Megarbane A, Jobard F, Debret $\mathrm{R}$, et al. A p.C217R mutation in fibulin-5 from cutis laxa patients is associated with incomplete extracellular matrix formation in a skin equivalent model. J Invest Dermatol 2008;128:1442-50.

23. Elahi E, Kalhor R, Banihosseini SS, Torabi N, Pour-Jafari H, Houshmand M, et al. Homozygous missense mutation in fibulin-5 in an Iranian autosomal recessive cutis laxa pedigree and associated haplotype. J Invest Dermatol 2006;126:1506-9.

24. Orriols M, Varona S, Marti-Pamies I, Galan M, Guadall A, Escudero JR, et al. Down-regulation of Fibulin-5 is associated with aortic dilation: role of inflammation and epigenetics. Cardiovasc Res 2016;110:431-42.

25. Cheng S, Lv H, Zhang W, Wang Z, Shi X, Liang W, et al. Adultonset demyelinating neuropathy associated with FBLN5 gene mutation. Clin Neuropathol 2017;36:171-7.

26. Stone EM, Braun TA, Russell SR, Kuehn MH, Lotery AJ, Moore $\mathrm{PA}$, et al. Missense variations in the fibulin 5 gene and age-related macular degeneration. N Engl J Med 2004;351:346-53.

27. Zengin MO, Karti O, Karahan E, Kusbeci T. An evaluation of the relationship between clinically unilateral pseudoexfoliation syndrome and age-related macular degeneration. Ophthalmic Surg Lasers Imaging Retina 2018;49:12-9.

28. Wirostko BM, Curtin K, Ritch R, Thomas S, Allen-Brady K, Smith KR, et al. Risk for exfoliation syndrome in women with pelvic organ prolapse: a Utah Project on Exfoliation Syndrome (UPEXS) study. JAMA Ophthalmol 2016;134:1255-62.

29. Gonen KA, Gonen T, Gumus B. Renal artery stenosis and abdominal aorta aneurysm in patients with pseudoexfoliation syndrome. Eye 2013;27:735-41.

30. Schumacher S, Schlotzer-Schrehardt U, Martus P, Lang W, Naumann GO. Pseudoexfoliation syndrome and aneurysms of the abdominal aorta. Lancet 2001;357:359-60.

31. Schlotzer-Schrehardt U, Hammer CM, Krysta AW, HofmannRummelt C, Pasutto F, Sasaki T, et al. LOXL1 deficiency in the 
lamina cribrosa as candidate susceptibility factor for a pseudoexfoliation-specific risk of glaucoma. Ophthalmology 2012;119:1832-43.

32. Want A, Gillespie SR, Wang Z, Gordon R, Iomini C, Ritch R, et al. Autophagy and mitochondrial dysfunction in tenon fibroblasts from exfoliation glaucoma patients. PLoS ONE 2016;11: e0157404.

33. Padhy B, Hayat B, Nanda GG, Mohanty PP, Alone DP. Pseudoexfoliation and Alzheimer's associated CLU risk variant, rs2279590, lies within an enhancer element and regulates CLU, EPHX2 and PTK2B gene expression. Hum Mol Genet 2017;26:4519-29.

34. Perez-Rico C, Pascual G, Sotomayor S, Asunsolo A, Cifuentes A, Garcia-Honduvilla N, et al. Elastin development-associated extracellular matrix constituents of subepithelial connective tissue in human pterygium. Invest Ophthalmol Vis Sci 2014;55:6309-18.

35. Ren T, Lin S, Wang Z, Shang A. Differential proteomics analysis of low- and high-grade of astrocytoma using iTRAQ quantification. Onco Targets Ther 2016;9:5883-95.

36. Yanagisawa H, Schluterman MK, Brekken RA. Fibulin-5, an integrin-binding matricellular protein: its function in development and disease. J Cell Commun Signal 2009;3:337-47.

37. Lotery AJ, Baas D, Ridley C, Jones RP, Klaver CC, Stone E, et al. Reduced secretion of fibulin 5 in age-related macular degeneration and cutis laxa. Hum Mutat 2006;27:568-74.

38. Markova D, Zou Y, Ringpfeil F, Sasaki T, Kostka G, Timpl R, et al. Genetic heterogeneity of cutis laxa: a heterozygous tandem duplication within the fibulin-5 (FBLN5) gene. Am J Hum Genet 2003;72:998-1004.

39. Callewaert B, Su CT, Van Damme T, Vlummens P, Malfait F, Vanakker $\mathrm{O}$, et al. Comprehensive clinical and molecular analysis of 12 families with type 1 recessive cutis laxa. Hum Mutat 2013;34:111-21.

40. Khadzhieva MB, Kamoeva SV, Chumachenko AG, Ivanova AV, Volodin IV, Vladimirov IS, et al. Fibulin-5 (FBLN5) gene polymorphism is associated with pelvic organ prolapse. Maturitas 2014;78:287-92.

41. Safka Brozkova D, Lassuthova P, Neupauerova J, Krutova M, Haberlova J, Stejskal D, et al. Czech family confirms the link between FBLN5 and Charcot-Marie-Tooth type 1 neuropathy. Brain 2013;136(Pt 7):e232.

42. Auer-Grumbach M, Weger M, Fink-Puches R, Papic L, Frohlich E, Auer-Grumbach P, et al. Fibulin-5 mutations link inherited neuropathies, age-related macular degeneration and hyperelastic skin. Brain 2011;134:1839-52.

43. Hu Q, Loeys BL, Coucke PJ, De Paepe A, Mecham RP, Choi J, et al. Fibulin-5 mutations: mechanisms of impaired elastic fiber formation in recessive cutis laxa. Hum Mol Genet 2006;15:3379-86.

44. Jones RP, Ridley C, Jowitt TA, Wang MC, Howard M, Bobola N, et al. Structural effects of fibulin 5 missense mutations associated with age-related macular degeneration and cutis laxa. Invest Ophthalmol Vis Sci 2010;51:2356-62.

45. Kaur I, Rathi S, Chakrabarti S. Variations in TIMP3 are associated with age-related macular degeneration. Proc Natl Acad Sci USA 2010;107:E112-3.
46. Kozobolis VP, Detorakis ET, Tsilimbaris MK, Vlachonikolis IG, Tsambarlakis IC, Pallikaris IG. Correlation between agerelated macular degeneration and pseudoexfoliation syndrome in the population of Crete (Greece). Arch Ophthalmol 1999;117:664-9.

47. Pasutto F, Zenkel M, Hoja U, Berner D, Uebe S, Ferrazzi F, et al. Pseudoexfoliation syndrome-associated genetic variants affect transcription factor binding and alternative splicing of LOXL1. Nat Commun 2017;8:15466.

48. Hauser MA, Aboobakar IF, Liu Y, Miura S, Whigham BT, Challa P, et al. Genetic variants and cellular stressors associated with exfoliation syndrome modulate promoter activity of a lncRNA within the LOXL1 locus. Hum Mol Genet 2015;24:6552-63.

49. Mullins RF, Olvera MA, Clark AF, Stone EM. Fibulin-5 distribution in human eyes: relevance to age-related macular degeneration. Exp Eye Res 2007;84:378-80.

50. Takacs P, Nassiri M, Viciana A, Candiotti K, Fornoni A, Medina CA. Fibulin-5 expression is decreased in women with anterior vaginal wall prolapse. Int Urogynecol J Pelvic Floor Dysfunct 2009;20:207-11.

51. Zhou Y, Ling O, Bo L. Expression and significance of lysyl oxidase-like 1 and fibulin-5 in the cardinal ligament tissue of patients with pelvic floor dysfunction. J Biomed Res 2013;27:23-8.

52. Chen X, Song X, Yue W, Chen D, Yu J, Yao Z, et al. Fibulin-5 inhibits Wnt/beta-catenin signaling in lung cancer. Oncotarget 2015;6:15022-34

53. Shi XY, Wang L, Cao CH, Li ZY, Chen J, Li C. Effect of Fibulin5 on cell proliferation and invasion in human gastric cancer patients. Asian Pac J Trop Med 2014;7:787-91.

54. Schlotzer-Schrehardt U, Pasutto F, Sommer P, Hornstra I, Kruse FE, Naumann GO, et al. Genotype-correlated expression of lysyl oxidase-like 1 in ocular tissues of patients with pseudoexfoliation syndrome/glaucoma and normal patients. Am J Pathol 2008;173:1724-35.

55. Papadopoulou M, Papadaki H, Zolota V, Gartaganis SP. Immunohistochemical profiles of LOXL-1, FBN1, TGF-beta1, and COX-2 in pseudoexfoliation syndrome. Curr Eye Res 2017;42:880-9.

56. Zenkel M, Poschl E, von der Mark K, Hofmann-Rummelt C, Naumann GO, Kruse FE, et al. Differential gene expression in pseudoexfoliation syndrome. Invest Ophthalmol Vis Sci 2005;46:3742-52.

57. Schlotzer-Schrehardt U. Genetics and genomics of pseudoexfoliation syndrome/glaucoma. Middle East Afr $\mathrm{J}$ Ophthalmol 2011;18:30-6.

58. Choi J, Bergdahl A, Zheng Q, Starcher B, Yanagisawa H, Davis EC. Analysis of dermal elastic fibers in the absence of fibulin-5 reveals potential roles for fibulin-5 in elastic fiber assembly. Matrix Biol 2009;28:211-20.

59. Hirai M, Ohbayashi T, Horiguchi M, Okawa K, Hagiwara A, Chien KR, et al. Fibulin-5/DANCE has an elastogenic organizer activity that is abrogated by proteolytic cleavage in vivo. $\mathrm{J}$ Cell Biol 2007; 176:1061-71. 